\title{
A numerical study of a turbulent mixing layer and its generated noise
}

\author{
LI Dong, GUO Li, ZHANG Xing \& HE GuoWei*
}

The State Key Laboratory of Nonlinear Mechanics, Institute of Mechanics, Chinese Academy of Sciences, Beijing 100190, China

Received March 26, 2013; accepted April 15, 2013; published online May 3, 2013

\begin{abstract}
A direct numerical simulation of a turbulent mixing layer with the Reynolds number 500 and the convective Mach number 0.6 is performed and the results obtained are used to study the turbulent flow field and its generated noise. In the present simulation, the numerical techniques of absorbing buffer zones, artificial convection velocity and spatial filtering are used to achieve nonreflecting boundary conditions. The self-similarity is used to validate the present numerical simulations. The large-scale coherent structures are plotted together with the acoustic waves, which demonstrates the directivity of acoustic waves. The Lighthill's source and space-time correlations are further investigated. The main contributions to mixing noise are identified in terms of large-scale coherent structures, Lighthill's source and space-time correlations.
\end{abstract}

direct numerical simulation, mixing layer, space-time correlation, noise

PACS number(s): 47.11.Bc, 47.27.ek, 47.27.wj

Citation: $\quad$ Li D, Guo L, Zhang X, et al. A numerical study of a turbulent mixing layer and its generated noise. Sci China-Phys Mech Astron, 2013, 56: 1157-1164, doi: $10.1007 / \mathrm{s} 11433-013-5098-1$

\section{Introduction}

A mixing layer is the turbulent flow that forms between two uniform and parallel streams of different velocities. It is the simplest model for initial shear layer regions of jets and the flows past a splitter in wind tunnel with many engineering applications [1]. Mixing layers are often used to study turbulent mixing and turbulence-generated noise, since the free shear effects could be fully displayed without any other complexity. Mixing layers are characterized by their self-similar states and large-scale coherent structures. The instability wave theory of sound generation is closely associated with the evolution of coherent structures in turbulent flows: small disturbances are amplified into large-scale coherent structures via the instability mechanism of shear layers and become largescale structures that lose their coherence to generate acoustic waves.

*Corresponding author (email: hgw@lnm.imech.ac.cn)
Lighthill [2,3] proposes an acoustic analog to relate the far-field noise with the flow fields. In the Lighthill acoustic analogy, the Lighthill stress tensor is directly calculated from the flow fields and plays a role of sound sources. Therefore, the Lighthill stress tensors are conventionally used to study sound sources. Lighthill acoustic analogy implies that the sound power spectra are dependent on the space-time correlation of velocity fluctuations. This suggests that space-time correlations can be used to identify the contributions of flow structures to far-field noise. Different models were developed for space-time correlations [4-10]. Fleury et al. [11] experimentally measured the space-time correlations in jets using dual particle image velocimetry(dual PIV). Cavalieri et al. [12] numerically compared the noise-controlled mixing layers with uncontrolled ones for noise reduction. Comparisons of direct numerical simulation with large eddy simulation of the mixing layer were also performed [13]. Freund et al. [1417] investigated compressibility effects in a turbulent annular mixing layer and noise radiated by turbulent jets systemat- 
ically. Direct numerical simulations of noise generated by a mixing layer with shock-cell structures were performed to investigate noise generated by the interaction between shock wave and vortex structures $[18,19]$.

In this paper, we use the direct numerical simulation (DNS) method to study the noise generated by the mixing layer and the related large-scale coherent structures, Lighthill's source and space-time correlations. In the second section, we will describe the numerical method. In the third section, we will present our numerical results and the final section is devoted to the summary.

\section{Numerical method}

\subsection{Governing equations}

The fluid is assumed to be a perfect gas with constant specific heat. In this case, the three-dimensional compressible Navier-Stokes equations can be written in the nondimensional form of

$$
\frac{\partial \boldsymbol{U}}{\partial t}+\frac{\partial \boldsymbol{E}}{\partial x}+\frac{\partial \boldsymbol{F}}{\partial y}+\frac{\partial \boldsymbol{G}}{\partial z}=\boldsymbol{H}
$$

where the capital vectors $\boldsymbol{U}, \boldsymbol{E}, \boldsymbol{F}, \boldsymbol{G}$ and $\boldsymbol{H}$ represent

$$
\begin{gathered}
\boldsymbol{U}=\left(\begin{array}{c}
\rho \\
\rho u_{x} \\
\rho u_{y} \\
\rho u_{z} \\
\rho e_{t}
\end{array}\right), \quad \boldsymbol{E}=\left(\begin{array}{c}
\rho u_{x} \\
\rho u_{x} u_{x}+p \\
\rho u_{x} u_{y} \\
\rho u_{x} u_{z} \\
\left(\rho e_{t}+p\right) u_{x}
\end{array}\right), \\
\boldsymbol{F}=\left(\begin{array}{c}
\rho u_{y} \\
\rho u_{y} u_{x} \\
\rho u_{y} u_{y}+p \\
\rho u_{y} u_{z} \\
\left(\rho e_{t}+p\right) u_{y}
\end{array}\right), \quad \boldsymbol{G}=\left(\begin{array}{c}
\rho u_{z} \\
\rho u_{z} u_{x} \\
\rho u_{z} u_{y} \\
\rho u_{z} u_{z}+p \\
\left.\rho e_{t}+p\right) u_{z}
\end{array}\right), \\
\boldsymbol{H}=\left(\begin{array}{c}
0 \\
\frac{\partial \tau_{x x}}{\partial x}+\frac{\partial \tau_{x y}}{\partial y}+\frac{\partial \tau_{x z}}{\partial \tau_{z}} \partial \\
\frac{\partial \tau_{y x}}{\partial x}+\frac{\partial \tau_{y y}}{\partial y}+\frac{\partial \tau_{y z}}{\partial z} \\
\frac{\partial \tau_{z x}}{\partial x}+\frac{\partial \tau_{z y}}{\partial y}+\frac{\partial \tau_{z z}}{\partial z} \\
-\frac{\partial q_{i}}{\partial x_{i}}+\frac{\partial\left(u_{i} \tau_{j} j_{j}\right.}{\partial x_{j}}
\end{array}\right) .
\end{gathered}
$$

The non-dimensional variables are $\boldsymbol{x}=\boldsymbol{x}^{*} / \delta_{\omega}^{0 *}, \quad \boldsymbol{u}=$ $\boldsymbol{u}^{*} / a_{\infty}^{*}, \quad t=t^{*} a_{\infty}^{*} / \delta_{\omega}^{0 *}, \rho=\rho^{*} / \rho_{\infty}^{*}, \quad p=p^{*} /\left(\rho_{\infty}^{*} a_{\infty}^{* 2}\right), \quad T=$ $[1 /(\gamma-1)] T^{*} / T_{\infty}^{*}, \mu=\mu^{*} / \mu_{\infty}^{*}$. The superscript '*' denotes dimensional variables and the subscript ' $\infty$ ' denotes the variables associated with free-stream. The Reynolds number based on sound speed is $\operatorname{Re}_{a}=\left(\rho_{\infty}^{*} \delta_{\omega}^{0 *} a_{\infty}^{*}\right) / \mu_{\infty}^{*}$. $\delta_{\omega}^{0 *}$ is the dimensional vorticity thickness of the laminar shear layer at the inlet. The specific heat ratio $\gamma=c_{p}^{*} / c_{v}^{*}=1.4$ is constant and the specific inner energy $e^{*}=c_{v}^{*} T^{*}$ is non-dimensionalized as $e=T / \gamma$ taking note of the definition of non-dimensional temperature. The specific total energy is defined as the sum of specific inner energy and kinetic energy $e_{t}=T / \gamma+u_{i} u_{i} / 2$. Thus, the non-dimensional state equation of ideal gas is given below

$$
p=(\gamma-1) \rho e=\frac{\gamma-1}{\gamma} \rho T
$$

$$
=(\gamma-1)\left(\rho e_{t}-\frac{1}{2} \rho u_{i} u_{i}\right) .
$$

The non-dimensional viscosity obeys a power law in the form

$$
\begin{aligned}
\mu & =\left(T^{*} / T_{\infty}^{*}\right)^{n} \\
& =[(\gamma-1) T]^{n} .
\end{aligned}
$$

Here the exponent $n=0.666$. The viscous stress tensor is

$$
\tau_{i j}=\frac{\mu}{R e_{a}}\left(\frac{\partial u_{i}}{\partial x_{j}}+\frac{\partial u_{j}}{\partial x_{i}}\right)+\frac{\mu}{R e_{a}}\left(\mu_{B}-\frac{2}{3}\right) \frac{\partial u_{k}}{\partial x_{k}} \delta_{i j},
$$

with the ratio of bulk viscosity to the dynamic viscosity being $\mu_{\mathrm{B}}=0.6$. The Fourier law of heat conduction $q_{i}^{*}=$ $-k^{*}\left(\partial T^{*}\right) /\left(\partial x_{i}^{*}\right)$ is used to express the heat flux vector:

$$
\begin{aligned}
q_{i} & =-k^{*} \frac{\partial T^{*}}{\partial x_{i}^{*}} \frac{1}{\rho_{\infty}^{*} a_{\infty}^{* 3}}=-\frac{\mu}{\operatorname{Re}_{a} \operatorname{Pr}} \frac{1}{\gamma-1} \frac{\partial}{\partial x_{i}}\left(\frac{T^{*}}{T_{\infty}^{*}}\right) \\
& =-\frac{\mu}{\operatorname{Re}_{a} \operatorname{Pr}} \frac{\partial T}{\partial x_{i}},
\end{aligned}
$$

where the Prandtl number is defined as $\operatorname{Pr}=\left(c_{p}^{*} \mu^{*}\right) / k^{*}=0.7$ with $k^{*}$ being the dimensional coefficient of thermal conductivity. Now the eqs. (1), (3) and (6) are closed for the hydrodynamical and thermodynamical variables.

\subsection{Spatial and temporal discretization}

An optimized sixth order compact (OPT6) finite difference scheme with spectral-like resolution is used in the streamwise and normal directions and a Fourier spectral method is used in the spanwise direction. The method ensures the precisions and dissipations required by noise predicting. The details on the used method can be found in reference [20]. The present scheme on boundaries should be specially treated: a third order compact asymmetric scheme is used right at the boundaries, a fifth order compact asymmetric scheme is used at the next-to-boundary nodes and a sixth order compact symmetric scheme is used at the second next-to-boundary nodes. The skew-symmetric form for the nonlinear convection terms is used to control the aliasing errors [18,21]

$$
\frac{\partial\left(\rho u_{j} q\right)}{\partial x_{j}}=\frac{1}{2}\left[\frac{\partial\left(\rho u_{j} q\right)}{\partial x_{j}}+q \frac{\partial\left(\rho u_{j}\right)}{\partial x_{j}}+\left(\rho u_{j}\right) \frac{\partial q}{\partial x_{j}}\right] .
$$

A two-step, fourth-order low dissipation and dispersion Runge-Kutta scheme $[18,22]$ is used to advance in temporal direction.

$$
\begin{aligned}
& \frac{\mathrm{d} u}{\mathrm{~d} t}=f(t, u(t)), \\
& t_{i-1}=t^{n-1}+c_{i} \Delta t, \\
& w_{i}=\alpha_{i} w_{i-1}+\Delta t f\left(t_{i-1}, u_{i-1}\right), \\
& u_{i}=u_{i-1}+\beta_{i} w_{i} .
\end{aligned}
$$

The CFL number can be calculated from

$$
\mathrm{CFL}=\left[\frac{k_{x}\left(\left|u_{x}\right|+a+U_{c}\right)}{\Delta x}+\frac{k_{y}\left(\left|u_{y}\right|+a\right)}{\Delta y}+\frac{k_{z}\left(\left|u_{z}\right|+a\right)}{\Delta z}\right] \Delta t,
$$


where $k_{x}, k_{y}, k_{z}$ are the maximum scaled wave numbers that can be resolved in three directions. $U_{c}$ is the artificially added convection speed in outflow sponge.

\subsection{Boundary conditions}

For the purpose of sound calculation, the efficient nonreflecting boundary conditions are needed. We will use the absorbing buffer zones in combination with an artificial convection term and a spatial fitting method to conform the nonreflecting boundary. A combination of Thompson's boundary conditions [23,24] and the damping sponge zones [18] is used for the lower and upper boundaries. In the absorbing sponge, an artificial term is added to the Navier-Stokes equations

$$
\frac{\partial \boldsymbol{Q}}{\partial t}=\text { Navier-Stokes terms }-\sigma_{y}(y)\left(\boldsymbol{Q}-\boldsymbol{Q}_{\mathrm{ref}}\right) .
$$

Here, the sponge strength $\sigma_{y}(y)$ is chosen to be identically zero within the physical domains and takes a finite value at the boundaries and decays gradually to zero in the sponge zones. To minimize transients that may influent noise prediction, we solve the steady, two-dimensional, compressible boundary layer equations to give the initial flow field. The reference state is the same as the initial flows, which are laminar and steady. The outflow boundaries are required to avoid turbulence-generated acoustic reflection at the exit of physical domain. In meeting this goal, an explicit spatial filter is taken to dissipate disturbances and an artificial convection term is added to the right-hand-side of the Navier-Stokes equations.

$$
\frac{\partial \boldsymbol{Q}}{\partial t}=\text { Navier-Stokes terms }-U_{c}(x) \frac{\partial \boldsymbol{Q}}{\partial x}
$$

At the inlet, a disturbance is imposed on the steady states in the sponge zones. The inflow disturbance is added based on the linear stability theory. The single instability wave has a formation $\hat{Q}(y) \exp [\mathrm{i}(\alpha x+\beta z-\omega t)]$, where $\hat{Q}(y)$ is the eigenfunction in the normal direction, $\alpha$ and $\beta$ are the wave numbers in the streamwise and spanwise directions and $\omega$ is the temporal frequency of the instability wave. Given a large number of these instability waves, the disturbances grow quickly along the streamwise direction.

The computational meshes are plotted in Figure 1 in the streamwise and normal direction with a grid skip of 8 . The computational domain is taken as $130 \delta_{\omega}^{0}, 65 \delta_{\omega}^{0}, 5 \delta_{\omega}^{0}$ in streamwise, normal and spanwise directions, respectively. The grids in each direction are 704, 320, 48. The physical domain ranges from $20 \delta_{\omega}^{0}$ to $90 \delta_{\omega}^{0}$ in the streamwise direction and from $-10 \delta_{\omega}^{0}$ to $45 \delta_{\omega}^{0}$ in the normal direction. The meshes are dense in the mixing regime to resolve larger velocity gradients and small-scale vortex structures. The grids in the spanwise direction are uniformly distributed.

\section{Numerical results}

We perform a direct numerical simulation of the threedimensional compressible mixing layer. The numerical setup is described as follows: the lower stream has a Mach number 1.2 and the upper stream is set to be quiescent, which facilitates the observation of local acoustic waves in the coordinate systems fixed in the laboratory. The Reynolds number based on the velocity difference between the two streams and the inflow vorticity thickness is 500 . The temperature and pressure ratio of the two streams are unity. As the flows become statistically stationary, a total of 1800 flow fields with time interval $0.1 \delta_{\omega}^{0} / a_{\infty}$ are used to calculate the statistical quantities. The ensemble averaging is taken over the 1800 flow fields along the temporal and spanwise directions. The validations of current simulation are given briefly in the first subsection and local flow structures are analyzed in the second subsection. Results about noise generated by mixing layer, Lighthill's source and space-time correlations are also discussed.

\subsection{Self-similarity}

In this subsection, we will present our numerical results of the self-similar states of mixing layer to validate our numerical simulation. Figure 2 plots the contours of mean streamwise velocity $\left\langle u_{x}\right\rangle$ with their contour levels from 0.1 to 1.1 with each increment 0.1 . The thickness of mixing layer grows nearly linear along streamwise direction, which is consistent with the theoretical predictions. Figure 3 shows the rescaled

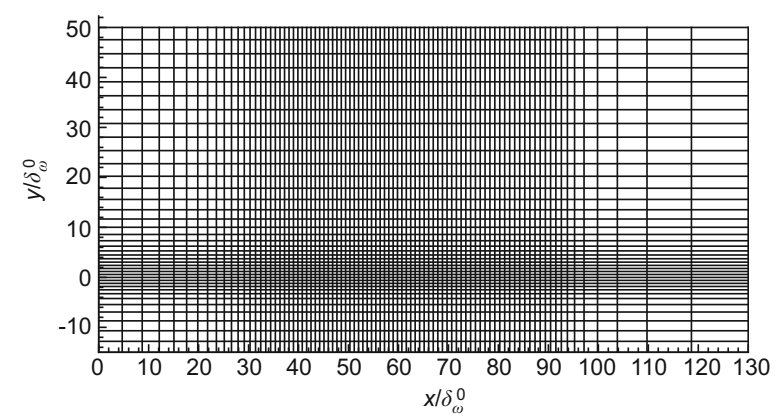

Figure 1 Sketch of the mesh set in the $x-y$ plane.

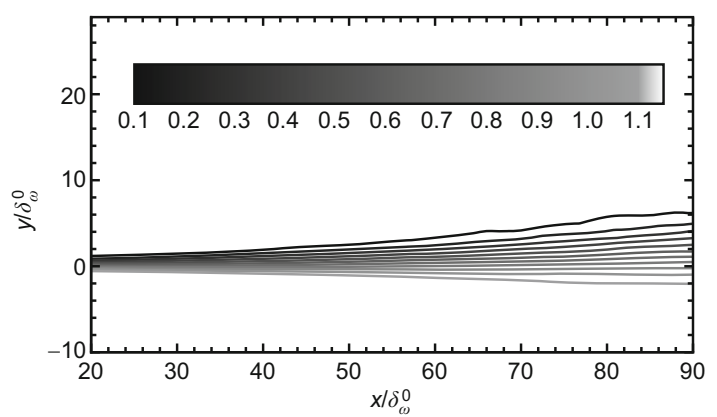

Figure 2 Mean streamwise velocity ranges from 0.1 to 1.1. The increment between contours is 0.1 . 


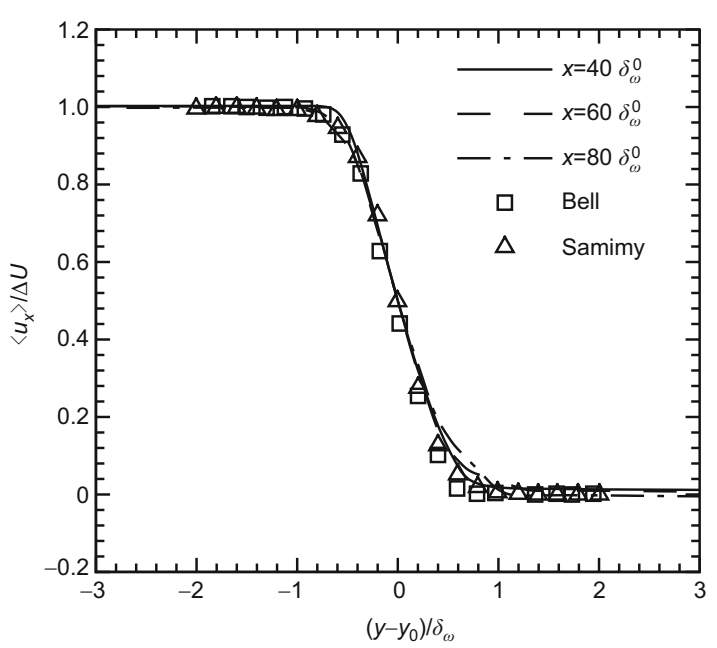

Figure 3 Self-similarity of velocity profiles. $\delta_{\omega}^{0}$ is the initial vorticity thickness. $\delta_{\omega}$ and $\Delta U$ are vorticity thickness and velocity difference across the shear layer respectively. $y_{0}$ is defined as the transverse location where $\left\langle u_{x}\right\rangle\left(y_{0}\right)=1 / 2 \Delta U$. Data of incompressible flow: ㅁ, Bell \& Mehta(1990) [25]; Data with convection Mach number 0.64: $\Delta$, Samimy \& Elliot(1990) [26].

mean streamwise velocities at three different streamwise locations. For comparison, we also plot the mean velocity profiles of incompressible flow from Bell \& Mehta [25] and the convection Mach number 0.64 one from Samimy \& Elliot
[26]. All of those curves collapse onto a single one, which validates our numerical simulation.

\subsection{Large-scale coherent structures and acoustic waves}

Figure 4 displays the near-field vortex structures and acoustic fields with time interval $\Delta t=2 \delta_{\omega}^{0} / a_{\infty}$. The local vortex structures are visualized using the colored contour of vorticity and the acoustic waves are visualized by dilatation. The dilatations are rendered in different levels of darkness while the positive (negative) dilatations beyond the thresholds are plotted as white (black).

Obviously, the vortex structures in the lower side (indicated by small red arrows in Figure 4) are convected to downstream quickly by mean flow while the convection effect is much smaller for the vortex in the upper side (indicated by small blue arrows in Figure 4). Meanwhile, the vortex structures in the mixing range rotate in anticlockwise direction with deformation and breakdown along the streamwise direction. The directivity of acoustic waves in the upper left corner can not be observed in Figure 4. The directional acoustic waves are obvious in the upper right corner and propagate with angles ranging from 30 to 40 degrees as sketched in Figure 4(a). It is also observed that these directional acoustic waves are mainly generated by the turbulent structures in up-

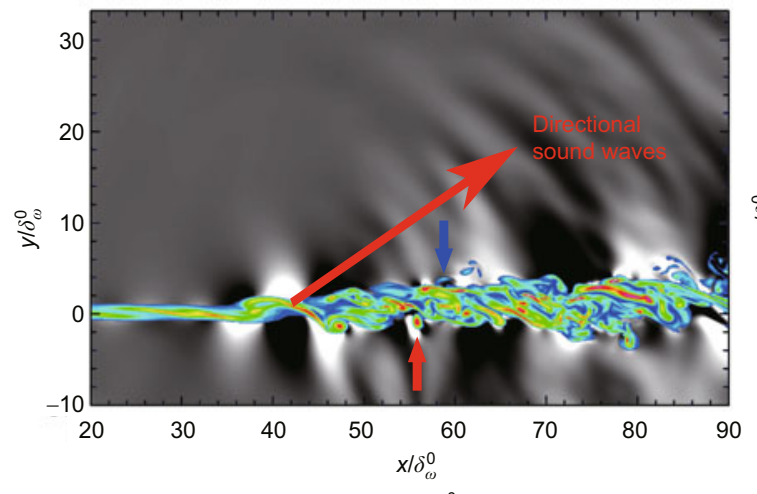

(a) $t=587 \delta_{\omega}^{0} / a_{\infty}$

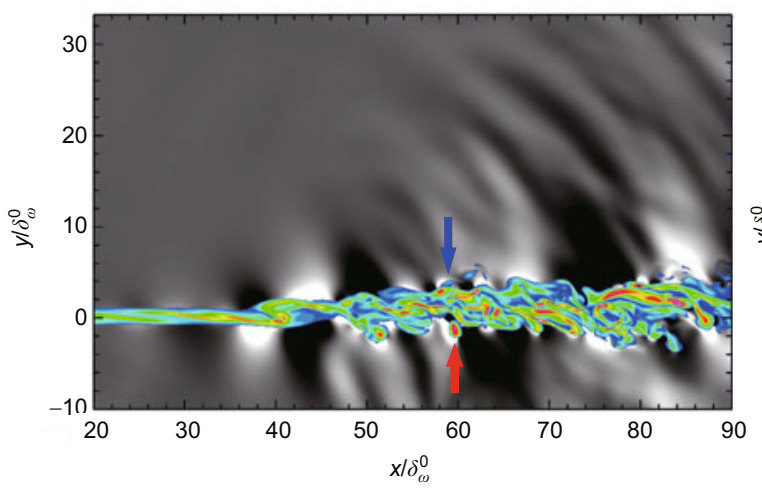

(c) $t=591 \delta_{\omega}^{0} / a_{\infty}$

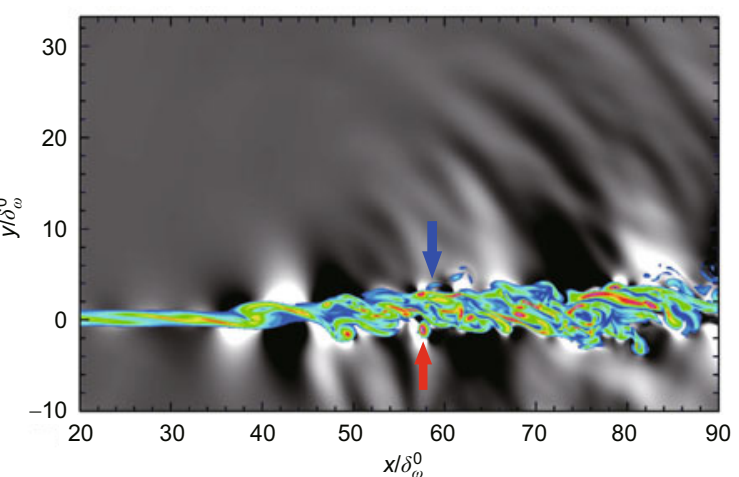

(b) $t=589 \delta_{\omega}^{0} / a_{\infty}$

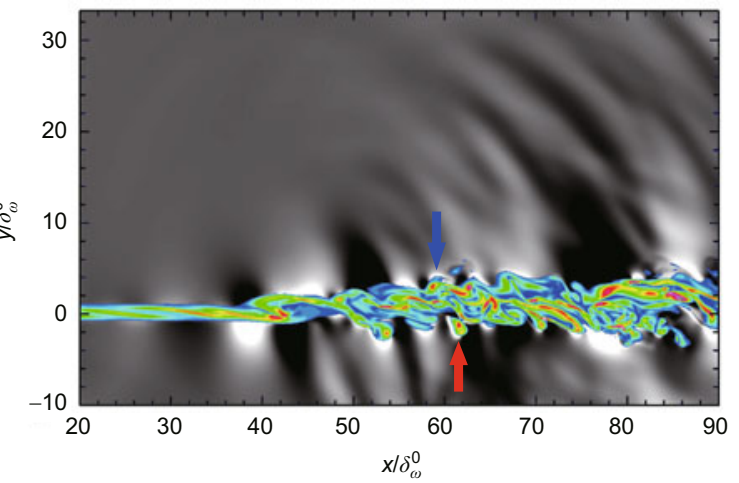

(d) $t=593 \delta_{\omega}^{0} / a_{\infty}$

Figure 4 Evolution of vortex structures and acoustic field. Colored contour of vorticity and grey scale map of dilatation are plotted together. (a)-(d) give the variations from $t=587 \delta_{\omega}^{0} / a_{\infty}$ to $t=593 \delta_{\omega}^{0} / a_{\infty}$. 
stream, which implies that the directional acoustic waves mainly come from the breaking down of large structures. The inlet disturbances in the form of instability waves radiate little noise. This implies that the inlet disturbances are acoustically quiet and the inlet conditions in present simulation are wellimposed. Furthermore, there is no reflected wave observed in the plots, which indicates that the non-reflecting boundaries work well.

\subsection{Turbulence-generated noise}

We extract the pressure signal at the plane $y=30 \delta_{\omega}^{0}$, where pressure fluctuations are used to represent acoustic signals. Figure 5 shows pressure intensity levels along the streamwise direction. The sound speed and density of free-stream are set as $a_{\infty}^{*}=340 \mathrm{~m} / \mathrm{s}$ and $\rho_{\infty}^{*}=1.29 \mathrm{~kg} / \mathrm{m}^{3}$ respectively. The dimensional pressure is $p^{*}=p \rho_{\infty}^{*} a_{\infty}^{* 2}$ and the sound pressure level is calculated from

$$
\mathrm{SPL}(\mathrm{dB})=10 \log _{10} \frac{\left\langle p^{* 2}\right\rangle}{p_{\mathrm{ref}}^{* 2}},
$$

where $p_{\text {ref }}^{*}=20 \mu \mathrm{Pa}$ is the lowest noise pressure level for the human ears. The location $x / \delta_{\omega}^{0} \simeq 35$ gives a turning point of sound intensity. The intensity of sound waves increases slowly before this point. After this point, the line goes up quickly.

We use the Kirchhoff integral formula [27] to calculate the noise at far-field. This formula utilizes the surface integrals, instead of volume integration, to calculate far-field noise: a planar surface is taken at $y=30 \delta_{\omega}^{0}$ and the pressure fluctuations on this surface are collected for the calculation of farfield noise. The effects of open Kirchhoff surface are limited to the upstream and downstream corners.

Figure 6 shows the far-field directional coefficients against the angle of observation. The origin point of observation is set at $\boldsymbol{x}=\left(55 \delta_{\omega}^{0}, 0\right)$ and the radial length is $100 \delta_{\omega}^{0}$. The increment of angle is $\theta=5^{\circ}$ with the starting angle $\theta=45^{\circ}$ and ending angle $\theta=135^{\circ}$. The directional coefficient is defined as:

$$
d(\theta)=\frac{\operatorname{SPL}(\theta)}{\operatorname{SPL}\left(\theta=135^{\circ}\right)} .
$$

The noise intensity goes up when the angle decreases. The directional coefficients are about 1.11 for $\theta=90^{\circ}$ and 1.28 for $\theta=45^{\circ}$.

A window function $f(t)$ is induced in the following form to get spectra of pressure fluctuations in the range $\left[t_{0}, t_{n}\right]$.

$$
f(t)=\frac{1}{2}\left[\tanh \left(5 \frac{t-t_{1}}{t_{1}-t_{0}}\right)+\tanh \left(5 \frac{t_{2}-t}{t_{n}-t_{2}}\right)\right],
$$

where $t_{1}$ and $t_{2}$ are set as the $5 \%$ and $95 \%$ points in time series. The pressure fluctuations $p^{\prime}(t)$ are multiplied by $f(t)$ and Fourier transformed to frequency space. Figure 7 gives the spanwise-averaged pressure spectra of two points. The fluctuations at point $\boldsymbol{x}=\left(60 \delta_{\omega}^{0}, 0\right)$ represent local turbulence

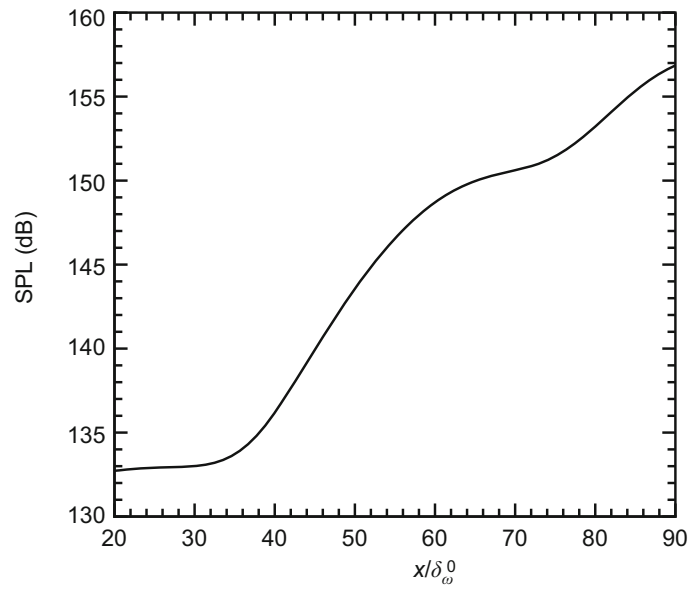

Figure 5 Distribution of sound pressure levels at the surface $y=30 \delta_{\omega}^{0}$ along the streamwise direction. The location $x / \delta_{\omega}^{0} \simeq 35$ gives the turnning point of sound intensity.

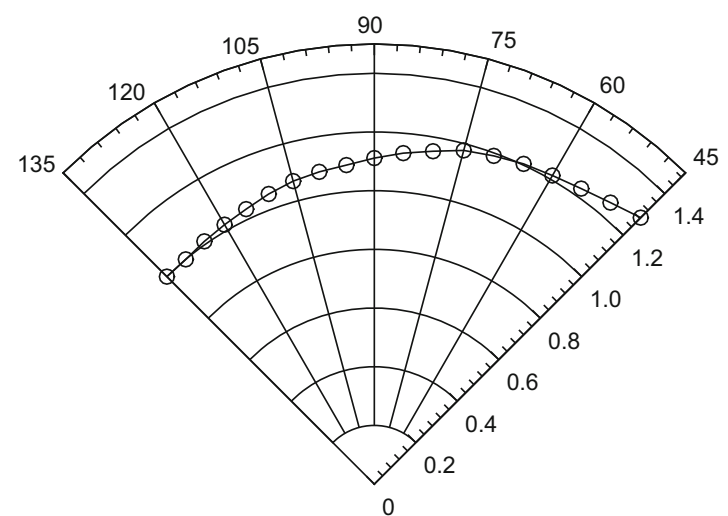

Figure 6 Directional coefficients of far-field sound pressure levels from the integration of Kirchhoff method. The observation is set as: origin of coordinates $\boldsymbol{x}=\left(55 \delta_{\omega}^{0}, 0\right)$; radial length $r=100 \delta_{\omega}^{0}$; increment of angle in anti-clockwise direction.

in the near field and the fluctuations at point $\boldsymbol{x}=\left(60 \delta_{\omega}^{0}, 40 \delta_{\omega}^{0}\right)$ can be regarded as acoustic signals in the far field. A slope $-5 / 3$ is also plotted to give the reference of decaying rate. There is no obvious power-law range expected in Figure 7 because of the low Reynolds number and limited spanwise length in the current simulation.

\subsection{Lighthill's source}

The so called Lighthill's equation can be expressed as follows $[2,3]$ :

$$
\left(\frac{\partial^{2}}{\partial t^{2}}-a_{\infty}^{2} \frac{\partial^{2}}{\partial x_{i} \partial x_{i}}\right)\left(\rho-\rho_{\infty}\right)=\frac{\partial^{2} T_{i j}}{\partial x_{i} \partial x_{j}} .
$$

The Lighthill stress tensor is

$$
T_{i j}=\rho u_{i} u_{j}+\left[\left(p-p_{\infty}\right)-a_{\infty}^{2}\left(\rho-\rho_{\infty}\right)\right] \delta_{i j}-\sigma_{i j}
$$

where $\delta_{i j}$ is the Kronecker delta and $\sigma_{i j}$ is the viscous stress tensor. The term

$$
\frac{\partial^{2} T_{i j}}{\partial x_{i} \partial x_{j}}
$$




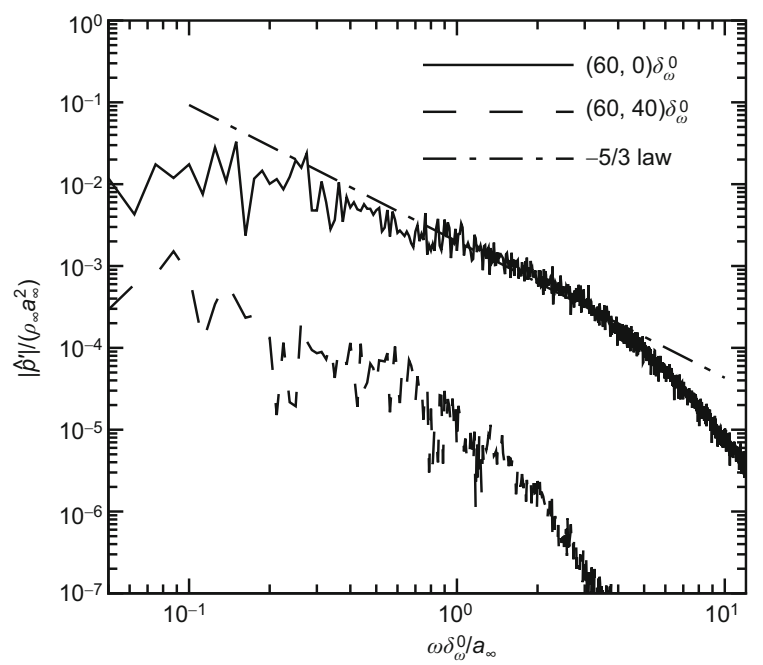

Figure 7 Spanwise-averaged pressure spectra. Solid line: $\boldsymbol{x}=\left(60 \delta_{\omega}^{0}, 0\right)$ in the near field; dashed line: $\boldsymbol{x}=\left(60 \delta_{\omega}^{0}, 40 \delta_{\omega}^{0}\right)$ in the acoustic field.

is regarded as Lighthill's source [16]. We will plot four components of Lighthill's source to identify their contributions to far-field noise. The viscous term is neglected because it's usually small compared with other terms. The selected components are $\partial^{2}\left(\rho u_{x} u_{x}\right) / \partial x^{2}, \partial^{2}\left(\rho u_{y} u_{y}\right) / \partial y^{2}, \partial^{2}\left(\rho u_{x} u_{y}\right) / \partial x \partial y$ and $\partial^{2}\left(p-a_{\infty}^{2} \rho\right) / \partial x^{2}$ corresponding to (b), (c), (d) and (e) in Figure 8, respectively. The local flow structures are also plotted as reference in Figure 8(a).

It is observed that the first component makes the largest contribution among the four components. The structures of the component in Figure 8(b) are similar to local vortex structures in Figure 8(a). The contour values in Figure 8(d) are smaller than the values in Figure 8(b) but larger than the term in Figure 8(c), which suggests that streamwise fluctuating velocity is the main sound source. The large structures in the downstream direction are abundant in Figures 8(b) and (d) and rare in Figure 8(c). Although the contour values in the upstream are small, the structures are usually more anisotropic, which could lead to apparent noise radiation. The term drawn in Figure 8(e) is responsible for the momentum transfer by pressure. This term is the smallest one compared with the other terms.

\subsection{Space-time correlations}

Intensity and spectrum of far-filed noise can be obtained by integrating the four-dimensional power spectral density tensor, which is associated with the space-time correlations of local flow velocities. Fleury et al. [11] gave the space-time second-order velocity autocorrelation function from dual PIV measurements. This autocorrelation function is defined as:

$$
R_{i i}(\boldsymbol{x}, \boldsymbol{\xi}, \tau)=\frac{\overline{u_{i}^{\prime}(\boldsymbol{x}, t) u_{i}^{\prime}(\boldsymbol{x}+\boldsymbol{\xi}, t+\tau)}}{\left[\bar{u}_{i}(\boldsymbol{x}) \bar{u}_{i}(\boldsymbol{x}+\boldsymbol{\xi})\right]},
$$

with $\bar{u}_{i}(\boldsymbol{x})$ being $\sqrt{\left\langle u_{i}^{\prime 2}(\boldsymbol{x}, t)\right\rangle}$ and $\bar{u}_{i}(\boldsymbol{x}+\boldsymbol{\xi})$ being $\sqrt{\left\langle u_{i}^{\prime 2}(\boldsymbol{x}+\boldsymbol{\xi}, t)\right\rangle}$.

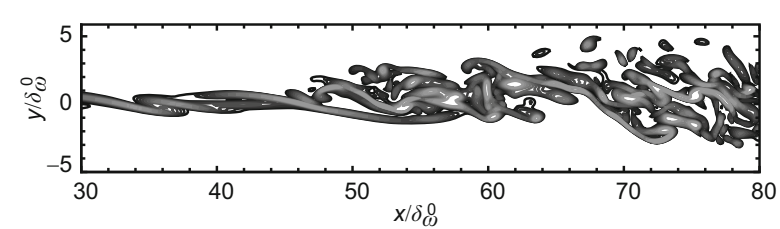

(a) Vorticity
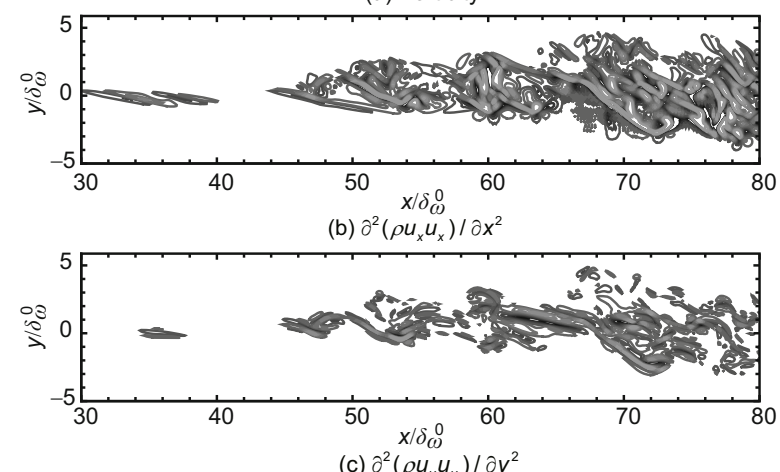

(c) $\partial^{2}\left(\rho u_{y} u_{y}\right) / \partial y^{2}$

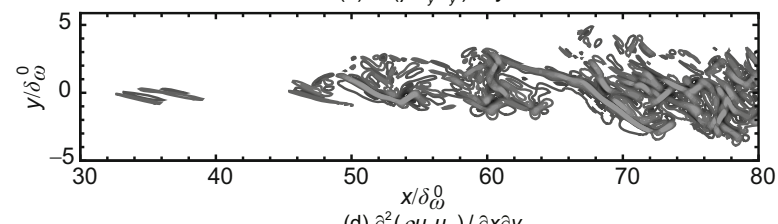

(d) $\partial^{2}\left(\rho u_{x} u_{y}\right) / \partial x \partial y$

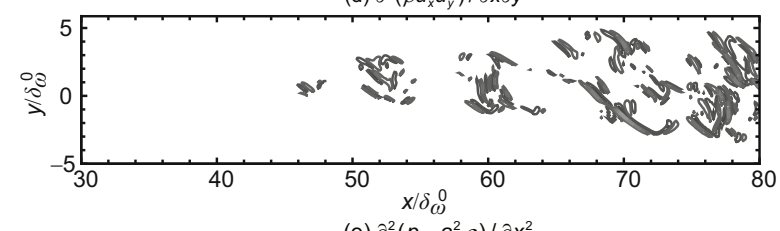

(e) $\partial^{2}\left(p-a_{\infty}^{2} \rho\right) / \partial x^{2}$

Figure 8 Contour plot of local vortex structures and components of Lighthill's source at $t=617.7 \delta_{\omega}^{0} / a_{\infty}$. (a): vorticity of fluid field; (b)-(e): The contour lines for Lighthill's source with a rendering range $[-1.0,1.0]$. The maximum contour values of (b), (c) and (d) exceed this range: (b) $[-7.4,10]$; (c) $[-2.8,1.5]$; (d) $[-5.2,3.8]$; (e) $[-0.89,0.94]$.

Figure 9 shows the spanwise-averaged space correlation. The correlation levels are $0.8,0.6,0.4,0.2,0.05,-0.05$ and -0.1 , the same levels that are plotted in the experimental measurements [11]. The contour lines are stretched and the stretched lines are more obvious for small iso-correlation levels. The negative correlation levels exist and their values are not ignorable, which is in agreement with the experiments [11].

Figure 10 plots the spanwise-averaged streamwise velocity correlation at point $\boldsymbol{x}=\left(60 \delta_{\omega}^{0}, 0\right)$ with spatial separation along the streamwise direction. The contour lines with larger values have the shape of ellipse. Negative values of correlation contour are also observed in the corner.

Figure 11 shows the integral scales of directional correlations for $R_{11}(\boldsymbol{x}, \boldsymbol{\xi}, \tau)$ at point $\boldsymbol{x}=\left(60 \delta_{\omega}^{0}, 0\right)$ with spatial separation along the streamwise direction [28]:

$$
L(\alpha)=\int_{\xi_{x} / \tau=\tan \alpha} \sqrt{1+\tan ^{2} \alpha} R_{11}\left(x, \xi_{x}, \tau\right) \mathrm{d} \tau .
$$

The directional correlation is calculated from the value of space-time correlation along a straight line $\xi_{x} / \tau=\tan \alpha$ with $0 \leqslant \alpha \leqslant \pi$. The integral scale of directional correlation gives 
the decorrelation length scale of the space-time correlation along a given direction. The position of its peak corresponds to the convection velocity at $\boldsymbol{x}=\left(60 \delta_{\omega}^{0}, 0\right)$.

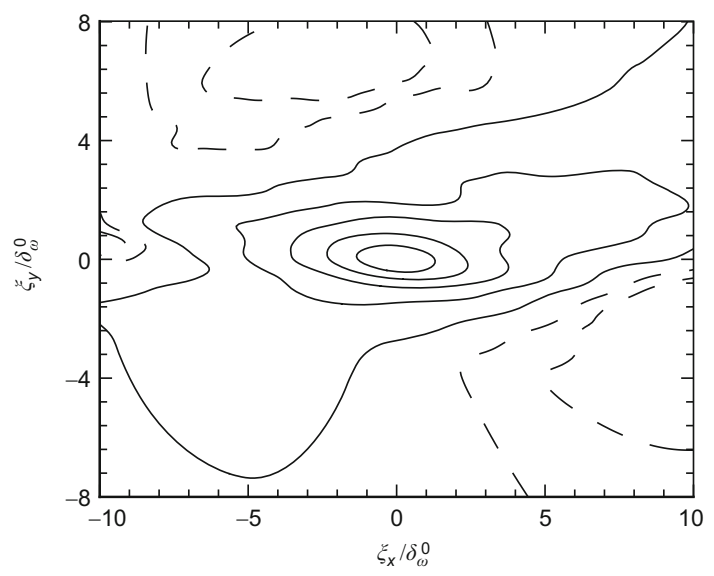

Figure 9 Spanwise-averaged space correlation $R_{11}(x, \xi, \tau)$ with $x=\left(60 \delta_{\omega}^{0}\right.$, $0)$ and $\tau=0$. The correlation levels are $0.8,0.6,0.4,0.2,0.05,-0.05$ and -0.1 with solid lines for positive values and dashed lines for negative values.

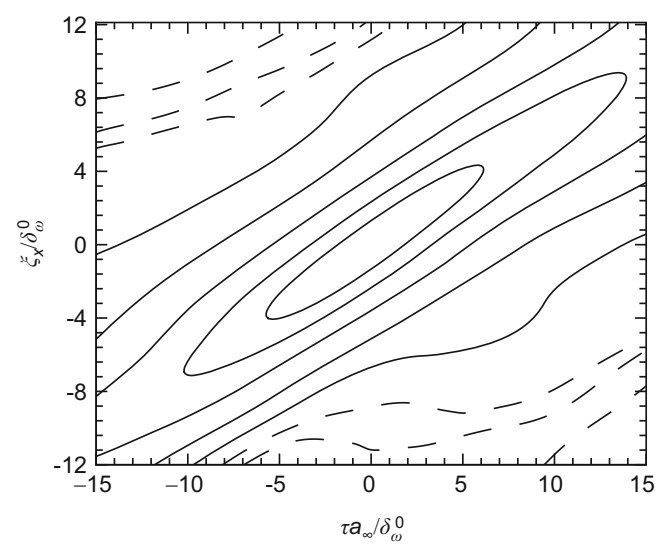

Figure 10 Spanwise-averaged space-time correlation $R_{11}(x, \xi, \tau)$ with $x=$ $\left(60 \delta_{\omega}^{0}, 0\right)$. The correlation levels are $0.8,0.6,0.4,0.2,0.05,-0.05,-0.1$ and -0.2 with solid lines for positive values and dashed lines for negative values.

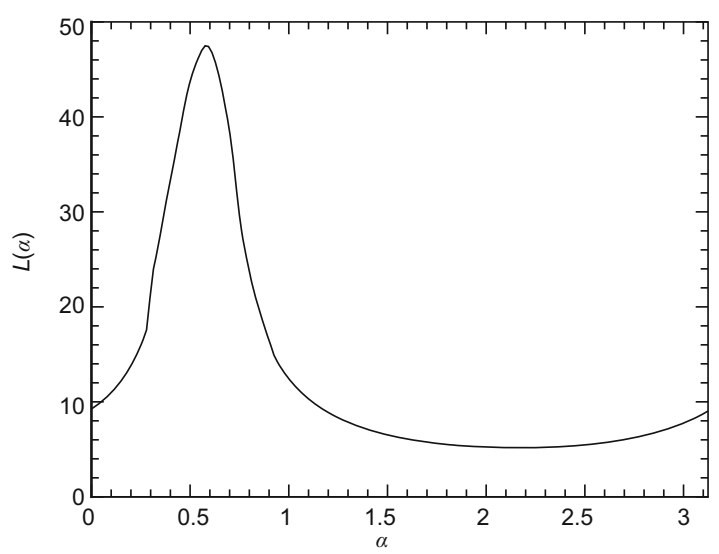

Figure 11 The integral scales of the directional correlations for $R_{11}(x, \xi, \tau)$ with $\boldsymbol{x}=\left(60 \delta_{\omega}^{0}, 0\right)$.

\section{Conclusions}

We perform a direct numerical simulation of spatially developing three-dimensional compressible mixing layer. The linear growth of mixing layer thickness and self-similar states are obtained from the present simulation, which validates the present numerical results. We directly calculate the near-field noise and use the open Kirchhoff surface integration to compute the far-field noise. It is observed that there exists a turning point where the SPL increases from a plateau to a slope. We further find that the upper structures in the mixing layer mainly undergo distortion while the lower structures are convected downstream with distortion. We compare the components of Lighthill's source in the near field: the streamwise fluctuating velocity makes larger contributions while the component responsible for momentum transfer by pressure makes smaller contribution. In the present study, the secondorder space-time autocorrelation functions are calculated and compared with experimental measurements and the geometries of iso-correlation lines are discussed.

The authors would like to thank Prof. CALVIN C. M. Lui for his support in numerical simulation. This work was supported by the National Natural Science Foundation of China (Grant Nos. 11232011 and 11021262)and the National Basic Research Program of China (Grant No. 2013CB834100) (Nonlinear science).

1 Colonius T, Lele S K, Moin P. Sound generation in a mixing layer. J Fluid Mech, 1997, 330: 375-409

2 Lighthill M J. On sound generated aerodynamically.1. General theory. Proc R Soc Lond A-Math Phys Sci, 1952, 211: 564-587

3 Lighthill M J. On sound generated aerodynamically.2. Turbulence as a source of sound. Proc R Soc Lond A-Math Phys Sci, 1954, 222: 1-32

4 Taylor G I. The spectrum of turbulence. Proc R Soc Lond A-Math Phys Sci, 1938, 164: 476-490

5 Kraichnan R H. The structure of isotropic turbulence at very high Reynolds numbers. J Fluid Mech, 1959, 5: 497-543

6 Kraichnan R H. Kolmogorov hypotheses and Eulerian turbulence theory. Phys Fluids, 1964, 7: 1723-1734

7 He G W, Zhang J B. Elliptic model for space-time correlations in turbulent shear flows. Phys Rev E, 2006, 73: 055303

8 He G W, Wang M, Lele S K. On the computation of space-time correlations by large-eddy simulation. Phys Fluids, 2004, 16: 3859-3867

9 Lee S, Lele S K, Moin P. Simulation of spatially evolving turbulence and the applicability of Taylors hypothesis in compressible flow. Phys Fluids A, 1992, 4: 1521-1530

10 Yao H D, He G W, Wang M, et al. Time correlations of pressure in isotropic turbulence. Phys Fluids, 2008, 20: 025105

11 Fleury V, Bailly C, Jondeau E, et al. Space-time correlations in two subsonic jets using dual particle image velocimetry measurements. AIAA J, 2008, 46: 2498-2509

12 Cavalieri A V G, Jordan P, Gervals Y, et al. Intermittent sound generation and its control in a free-shear flow. Phys Fluids, 2010, 22: 115113

13 Bodony D J. Aeroacoustic prediction of turbulent free shear flows. Dissertation for the Doctoral Degree. Stanford: Stanford University, 2004

14 Freund J B, Lele S K, Moin P. Compressibility effects in a turbulent annular mixing layer. part 1 . Turbulence and growth rate. J Fluid Mech, 
2000, 421: 229-267

15 Freund J B, Moin P, Lele S K. Compressibility effects in a turbulent annular mixing layer. part 2. Mixing of a passive scalar. J Fluid Mech, 2000, 421: 269-292

16 Freund J B. Noise sources in a low-Reynolds-number turbulent jet at Mach 0.9. J Fluid Mech, 2001, 438: 277-305

17 Freund J B. Noise-source turbulence statistics and the noise from a Mach 0.9 jet. Phys Fluids, 2003, 15: 1788-1799

18 Lui C C M. A numerical investigation of shock-associated noise. Dissertation for the Doctoral Degree. Stanford: Stanford University, 2003

19 Schaupp C, Sesterhenn J, Friedrich R. On a method for direct numerical simulation of shear layer/compression wave interaction for aeroacoustic investigations. Comput Fluids, 2008, 37: 463-474

20 Lele S K. Compact finite-difference schemes with spectral-like resolution. J Comput Phys, 1992, 103: 16-42

21 Blaisdell G A, Spyropoulos E T, Qin J H. The effect of the formulation of nonlinear terms on aliasing errors in spectral methods. Appl Numer
Math, 1996, 21: 207-219

22 Stanescu D, Habashi W G. 2Nstorage low dissipation and dispersion Runge-Kutta schemes for computational acoustics. J Comput Phys, 1998, 143: 674-681

23 Thompson K W. Time-dependent boundary-conditions for hyperbolic systems. J Comput Phys, 1987, 68: 1-24

24 Thompson K W. Time-dependent boundary-conditions for hyperbolic systems.2. J Comput Phys, 1990, 89: 439-461

25 Bell J H, Mehta R D. Development of a two-stream mixing layer from tripped and untripped boundary layers. AIAA J, 1990, 28: 2034-2042

26 Samimy H, Elliot G S. Effect of compressibility on the characteristics of free shear layers. AIAA J, 1990, 28: 439-445

27 Freund J B, Lele S K, Moini P. Calculation of the radiated sound field using an open Kirchhoff surface. AIAA J, 1996, 34: 909-916

28 Guo L, Li D, Zhang X, et al. LES prediction of space-time correlations in turbulent shear flows. Acta Mech Sin, 2012, 28: 993-998 